\title{
Communicable Diseases
}

\section{National Congenital Rubella Surveillance Programme 1 July 1971-30 June 1984}

\author{
R W SMITHELLS, SHULA SHEPPARD, HELEN HOLZEL, ANN DICKSON
}

This is the fourth report of a series on the surveillance of congenital rubella by the National Congenital Rubella Surveillance Programme using methods previously described in the $B M \mathcal{F} .^{1.3} \mathrm{We}$ present data on 763 children (including nine co-twins, that is, nine pairs) classified by the Northern and Southern Registries of the National Congenital Rubella Surveillance Programme as cases of confirmed or suspected congenital rubella. The children were reported between 1 July 1971 and 30 June 1984, a period during which large and moderate rubella epidemic years were interspersed among non-epidemic years (figure). The same figure shows by year
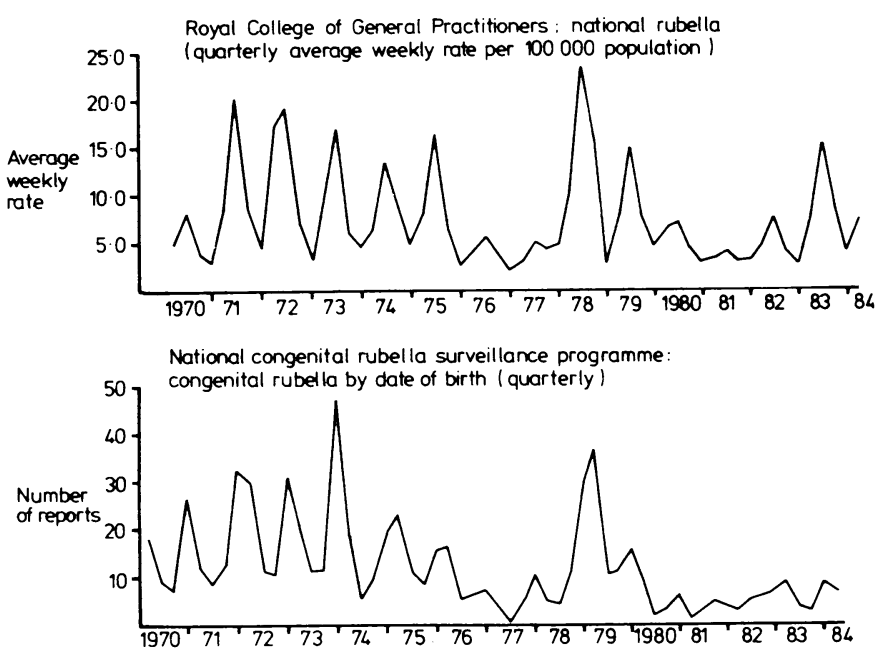

of birth the 667 children born during the same period and registered as confirmed or suspected congenital rubella, with the time scale advanced by six months to that of the data on the epidemics.

The number of children reported to the National Congenital Rubella Surveillance Programme and classified as cases of con-

University Department of Paediatrics, Leeds General Infirmary, Leeds LS2 9NS R W SMITHELLS, MD, FRCP, professor of paediatrics and child health SHULA SHEPPARD, MB, CHB, research fellow

Department of Microbiology, Hospital for Sick Children, London WC1N 3JH HELEN HOZEL, MD, MRCPATH, consultant microbiologist and honorary senior lecturer

ANN DICKSON, research assistant southern registry

Correspondence to: Dr N S Galbraith, PHLS Communicable Disease Surveillance Centre, 61 Colindale Avenue, London NW9 5EQ. firmed or suspected congenital rubella varied from year to year (table I); 1973 remains the year in which the largest number of children (89) was registered. Thereafter numbers dropped to 18 in 1977 , and then increased after the widespread rubella epidemic in 1978 and 1979 to 50 and 74 children respectively. The total for these two years, 124 children, includes a further 19 registrations since our last analysis, ${ }^{3}$ and underlines the essentially provisional nature of the more recent data. 1980 and 1981 were non-epidemic years and were followed by two years with moderate epidemics, although data available suggest that registrations in 1982 and 1983 may well be fewer than those for 1978 and 1979.

The clinical manifestations in 763 children, together with their pregnancy order and some relevant maternal history, are summarised in table II. Since the last report the additional registrations have been in respect of children born in 1977 or later, with two exceptions (one child born in 1975 and one born in 1976). Data for 1970-6 remain substantially unchanged and analyses for these years have been summarised.

\section{Maternal history}

Overall, 347 of the 732 mothers $(47 \%)$ gave a history of a rubella like illness in pregnancy; a further $108(15 \%)$ had been in contact with rubella but did not have a subsequent rubella like illness. No

TABLE I-Children with confirmed or suspected congenital rubella by year of birth

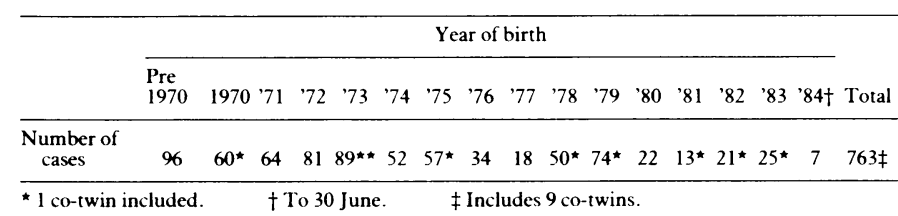

TABLE II-Clinical data for 763 confirmed/suspected cases of congenital rubella

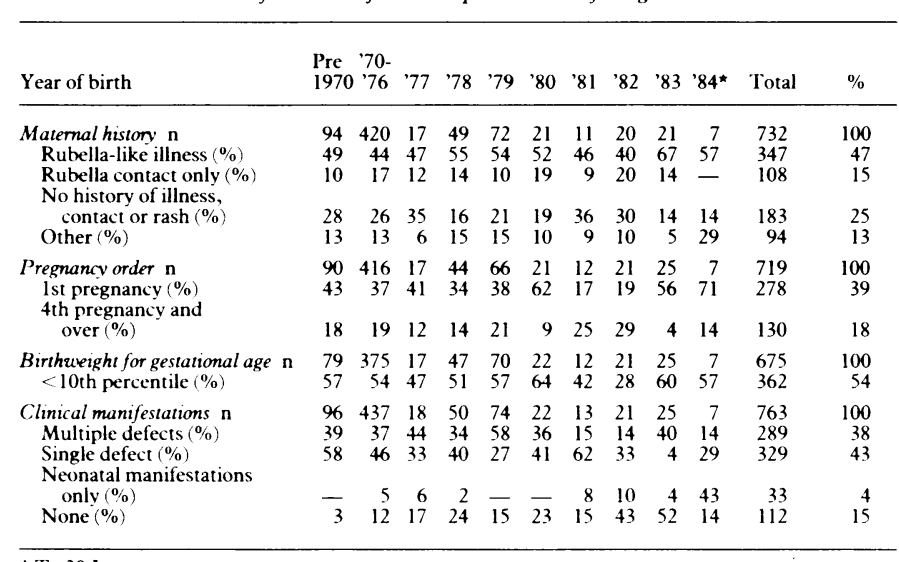

$\star$ To 30 June. 
fewer than 183 mothers (a quarter of the total) were apparently well throughout pregnancy. Symptoms and illnesses recorded for the remaining mothers include undiagnosed rash, sore throat, influenza ("other" in table II). In 1983 the highest proportion of mothers with a history of a rubella like illness in pregnancy $(67 \%)$ was reported; this figure probably reflects the early investigation, diagnosis, and notification of their infants.

\section{Pregnancy order}

Of the 719 children affected, $278(39 \%)$ were first born. The considerable variation seen in this proportion from year to year is particularly apparent in years when small numbers were reportedfor example, 1981 (17\%) and 1984 (71\%). Interpretation of data relating to pregnancy order may prove difficult because while the main thrust of the rubella vaccination policy in Britain is directed at girls before they reach childbearing years the policy now includes the ascertainment and protection of all susceptible women of childbearing age.

\section{Birthweight for gestational age}

Of those 675 children for whom both birthweight and gestational age have been recorded, $362(54 \%)$ fell below the 10 th percentile.

\section{Clinical manifestations}

Of the 763 children, $618(81 \%)$ had one or more defects $(38 \%$ multiple, $43 \%$ single). The remainder comprised a small group of 33 children $(4 \%)$ who had neonatal signs of a systemic infection and a larger group of 112 children (15\%) for whom there is laboratory evidence alone of a prenatal infection; they form the "at risk" group in whom rubella defects-in particular, a sensorineural hearing loss - may become apparent within a year or two. This may in part explain the relatively high proportion $(55 \%)$ of children born in 1982 and later who are assigned to this group. Follow up information is sought for all children registered in the programme, and forms the basis for re-assignment to another category where appropriate.

The proportion of the mothers of all the infants registered by the National Congenital Rubella Surveillance Programme and deemed to have been eligible for immunisation as schoolgirls-that is, women born in 1958 or later-still remains small $(7 \%)$.

\section{Impact of the rubella vaccination programme}

Rubella vaccine has now been available in Britain for 14 years, during which the principal target group has been schoolgirls aged 13 years or younger. Their immunisation alone could not be expected to make much impact on the incidence of congenital rubella in the first 10 years or so of the programme.

In any assessment of the vaccination programme it is essential to consider rubella associated terminations of pregnancy (particularly those associated with disease or contact) as well as cases of congenital rubella. Continued monitoring of these terminations and of congenital rubella cases is likely to give an indication of the effectiveness of the policy within the next five years. Crucial to this assessment will be the preservation of school medical and immunisation records. Without them, and at a distance of some 10 or more years, it may not be possible to distinguish from a mother's history between rubella and BCG immunisations, which are carried out in similar age groups.

\section{References}

1 Anonymous. National Congenital Rubella Surveillance Programme. Br Med f 1979;ii:396-7. 2 Anonymous. National Congenital Rubella Surveillance Programme. Br Med f 1981;282:324. 3 Smithells RW, Sheppard S, Marshall WC, Milton A. National Congenital Rubella Surveillance Programme 1971-81. BrMed f 1982;285:1363.

\section{CDSC Report}

\section{Outbreak of poliomyelitis in Finland}

Finland, which instituted a strong programme of poliomyelitis immunisation in the early 1960s, has achieved coverage of more than $90 \%$ of the population ( $98 \%$ of those under 1 year). No cases of poliomyelitis have been reported during the past two decades. The immunisation programme is based on the use of six doses of inactivated poliovirus vaccine given at the ages of 5 months, 6 months, 2 years, 6 years, 11 years, and 16 years respectively. Men are given an additional dose of vaccine during military service at 20 years. The vaccine is produced by RIT Laboratories, Belgium.

In mid October 1984 a 6 year old boy with mild meningitis was shown to be excreting poliovirus type 3 . He had been fully up to date with his vaccination schedule, having had three doses. Investigation of healthy contacts and other healthy people in his neighbourhood close to Helsinki showed the virus to be widespread. This was the first evidence of indigenous poliovirus circulation in the past 20 years in Finland. Six isolates sent to a laboratory in the Netherlands for strain analysis were all found to be "not vaccine like."

In November/December 1984 poliovirus type 3 was isolated from five more patients with paralytic disease typical of poliomyelitis. One of the patients was a 31 year old pregnant woman who had had no poliovirus vaccinations; another was a 33 year old man with
Hodgkin's disease who had had only a single dose of vaccine about 20 years ago. Two other patients were boys aged 17 and 12 years who had each received five doses of the regular vaccine. The 17 year old boy, who had respiratory paralysis, died as a result of an anaesthetic given for a repair of a tracheostomy. A sixth case was in a 4 year old boy with mild encephalitis and paralysis (now fully recovered) who had had two doses of the vaccine. Another four cases still being investigated have clinical paralytic disease suggestive of poliomyelitis; the date of onset of one of these cases was August 1984. Shedding of poliovirus type 3 into faeces or the throat, or both, has been found in 100 healthy people, mostly children, out of about 700 studied so far. These people were contacts of the first case or contacts of known excreters of the virus. Like the patients, the carriers of the virus were from different locations throughout Finland. Poliovirus type 3 has also been isolated from all eight sewage water specimens collected at eight different sites in the Helsinki district, and so far from 12 specimens from 21 cities throughout the country, although there is now some evidence that circulation of poliovirus type 3 is slowing down. Previously, until 1981, sewage waters in Helsinki had been regularly screened for viruses, and not a single poliovirus had been isolated from 408 